\title{
Study on the Pictograph of Dongba Script in Lijiang Area
}

\author{
Yin Zhou ${ }^{1,2}$ \\ ${ }^{1}$ Humanities College, Chongqing Jiaotong University, Chongqing, China \\ ${ }^{2}$ Chinese History Postdoctoral Research Station, Southwest University, Chongqing, China \\ Email: zhouyin2008@126.com
}

How to cite this paper: Zhou, Y. (2018) Study on the Pictograph of Dongba Script in Lijiang Area. Open Journal of Social Sciences, 6, 122-130.

https://doi.org/10.4236/jss.2018.63008

Received: February 2, 2018

Accepted: March 16, 2018

Published: March 19, 2018

Copyright (c) 2018 by author and Scientific Research Publishing Inc. This work is licensed under the Creative Commons Attribution International License (CC BY 4.0).

http://creativecommons.org/licenses/by/4.0/

\begin{abstract}
Lijiang, located in the northwest of Yunnan province, is famous for its dongba script. Dongba script is an important pictograph, which is still in use in border area between Yunnan and Sichuan. This article researches on the pictograph of dongba script, and focuses on the classification of pictograph of dongba script. The study of dongba script helps to study the origin and development of script.
\end{abstract}

\section{Keywords}

Dongba Script, Pictograph, Lijiang Area

\section{Introduction}

Dongba culture is the excellent traditional culture of ethnic of Naxi. Lijiang, located in the northwest of Yunnan province, is the main settlement of Naxi [1]. Lijiang dongba culture is prosperous on the history, and there have been many famous dongba. See Figure 1. They leave a lot of beautifully decorated, elegant dongba scriptures [2]. The American scholar Joseph Charles Francis Rock has been collecting dongba manuscripts in Sichuan and Yunnan Provinces. See Figure 2. Most of the dongba manuscripts are collected from Lijiang region and are mainly kept in the Harvard-Yenching Institute [3].

The dongba manuscripts in Lijiang region center in the Dayan Town, including the surrounding Baisha Town, Tai'an Town, Huangshan Town, Qihe Town and other places [2]. We will study the dongba script in these areas. The materials mainly come from < An Annotated Collection of Naxi Dongba Manuscripts $>[4]$, which is the biggest collection of dongba manuscripts, covering almost all areas of Lijiang dongba manuscripts. We selected the dongba manuscripts in Dayan Town, Huangshan Town, Qihe Town to study. And the 


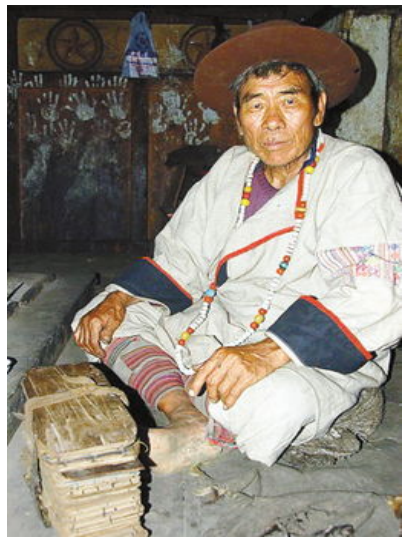

Figure 1. Naxi priest Dongba.

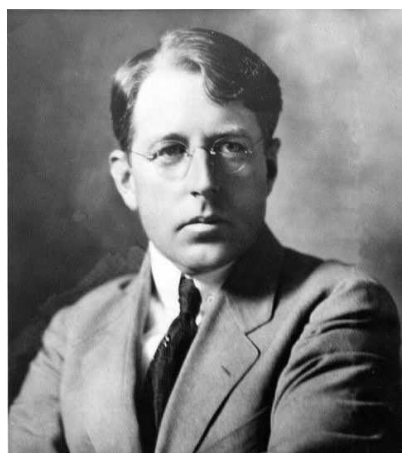

Figure 2. Joseph Charles Francis Rock.

materials quoted from the $<$ An Annotated Collection of Naxi Dongba Manuscript $>$ will be marked the volume and the page. "88.191" means that the materials come from volume 88, page 191 .

But $<$ An Annotated Collection of Naxi Dongba Manuscripts $>$ misses famous dongba manuscripts in Baisha area. We are lucky to find them in $<$ Naxi Dongba Manuscripts Collected by Harvard-Yenching Institute > [5], which is a great collection of dongba manuscripts. These materials come from Harvard-Yenching Institute, and most of them are collected by Joseph Charles Francis Rock. The materials quoted from the < Naxi Dongba manuscripts collected by Harvard-Yenching Institute > will be marked the volume and the page. "H4.471" means the materials come from volume 4, page 471.

\section{The Pictograph Character of Dongba Script}

Dongba script is an original but mature ideogram, and the total number of dongba script is about one thousand and four hundred [6]. During its long history of development, it evolves out some other creating methods. According to the creating method of dongba script, we can divide dongba script into four categories: pictograph character, indicative character, associative character, and picto-phonetic character. And the majority of them are pictograph character, so we focus on the pictograph character of dongba script. See Figure 3. 


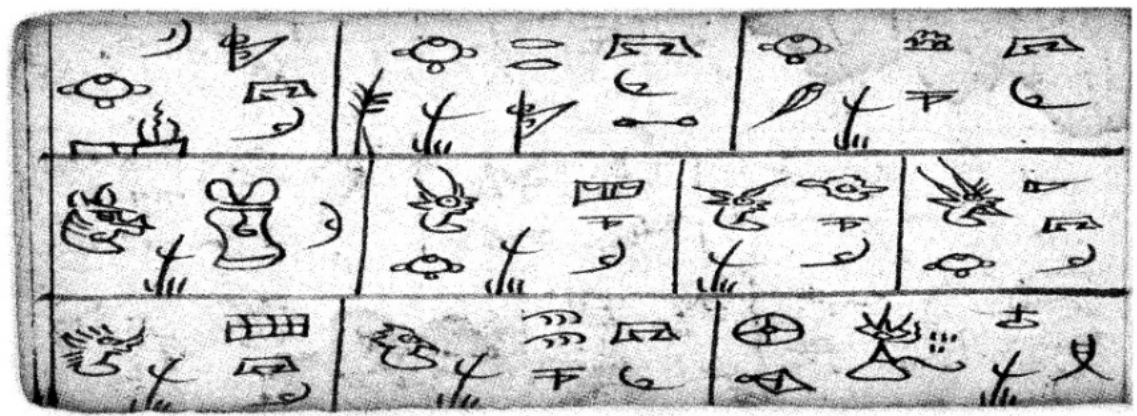

Figure 3. The Dongba Manuscript in Lijiang.

According to our study, the pictograph of dongba script can be divided into dependent pictograph and compound pictograph [6]. Now we separately study the dongba pictograph in Lijiang area on these two aspects.

\section{Dependent Pictograph of Dongba Script in Lijiang}

Dependent pictograph means the script cannot be divided, no matter writing out the whole image or the partial image. According to the pattern the object being taken, dependent pictograph of dongba script can be divided into two parts: the overall pictograph and partial pictograph.

\subsection{Overall Pictograph}

Overall pictograph writes out the overall image of object, the meaning expressed by the script equal to the word's meaning. Overall pictograph in Lijiang area involves a wide range on the meaning category, and the number of the dongba form is large.

\subsubsection{Overall Pictograph on Astronomy and Geography}

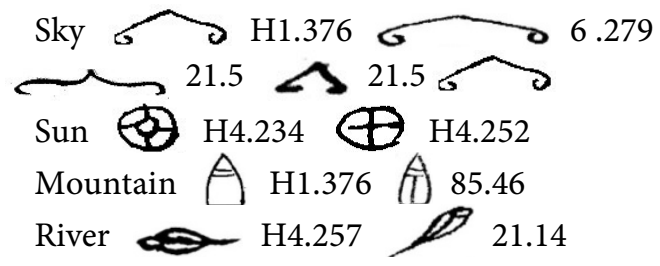

The scripts of the astronomy and geography are created according to the overall shape of the objects. When you see the script, you get the meaning at first glance.

It should be noted that the font $\bigoplus\left[\mathrm{ni}^{33} \mathrm{me}^{33}\right]$ is rarely used to express the original meaning of the "sun", but often used together with $\left[\mathrm{kv}^{33}\right]$ to express the meaning of "sunset" $\left[\mathrm{n}^{\mathrm{i}}{ }^{33} \mathrm{me}^{33} \mathrm{gv}^{21}\right]$, then borrowed to express the meaning of "west" $\left[\mathrm{ni}^{33} \mathrm{me}^{33} \mathrm{gv}^{21}\right]$. There is the example of just using $\Theta$ to express "west".

The meaning of the scripture in Figure 4 is: "Quartet: east, west, north, and south."

Here $\circledast$ expresses the meaning of "east", and $\$$ expresses the meaning of "west". 


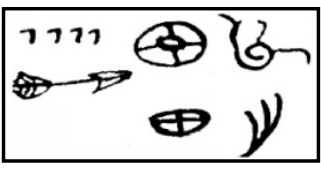

Figure 4. 88.191.

Though the original meaning of $\bigoplus$ and $\notin$ is "sun", the two fonts also have different meanings to a certain extent. $\$$ is often used together with [thv33] to express the meaning of "east" [ni $\mathrm{i}^{33} \mathrm{me}^{33}$ thv33], and $\boldsymbol{F}$ often used together with $\bigcirc\left[\mathrm{kv}^{33}\right]$ to express the meaning of "west". So dongba can just use $\notin$ to express "east", and use $\circledast$ to express "west".

\subsubsection{Overall Pictograph on Plants, Animals and Human Body}

The scripts of the plants, animals and human body are created according to the overall shape of the objects.

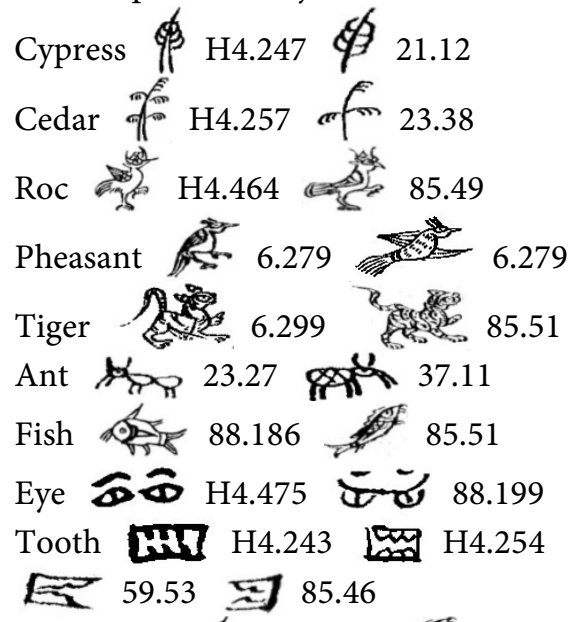

The fonts are just like a picture of "fish", writing the whole shape of the fish. The fonts $\mathbf{H D}$ and $\sqrt{\mathbf{S}}$ are just what the tooth looks like.

\subsubsection{Overall Pictograph on Diet and Living}

The scripts of the diet and living are created according to the overall shape of the objects.

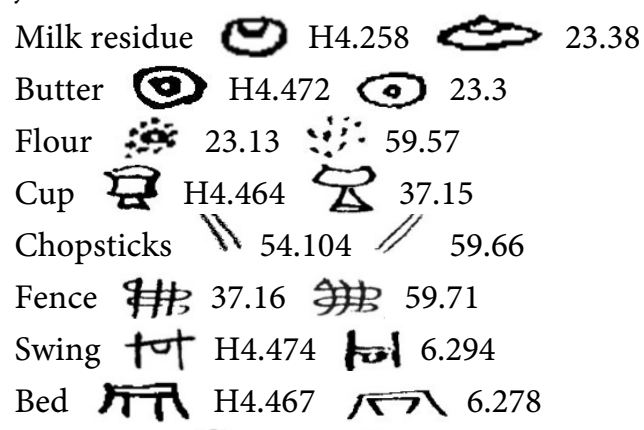

The fonts and and like a picture of "flour". The feature of the flour is a lot of small solid particles, so it uses many dots to represent the meaning of flour. 


\subsubsection{Overall Pictograph on Clothing and Appliances}

The scripts of the clothing and appliances are created according to the overall shape of the objects.
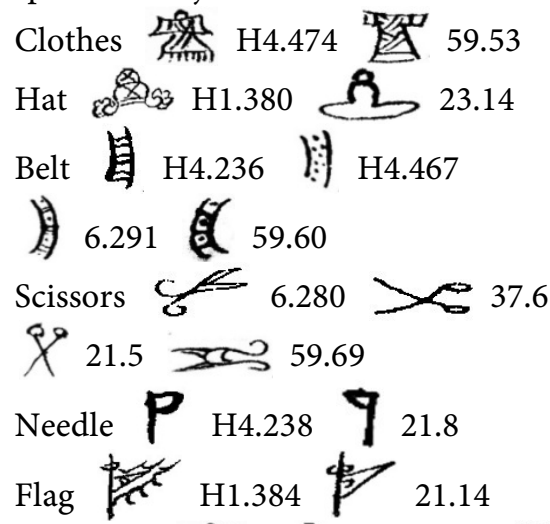

The fonts 㒄秎 to express the meaning, you can understand them at first glance.

\subsection{Partial Pictograph}

Partial pictograph writes out the partial image of the object, and the information expressed by the script is less than the meaning of the word. The distribution area of partial pictograph script in Lijiang dongba is smaller than the overall pictograph, and it is mainly distributed in plants, birds and animals. However, the diversity of Lijiang's partial pictograph is more plentiful than the overall pictograph.

\subsubsection{Partial Pictograph on Plant}

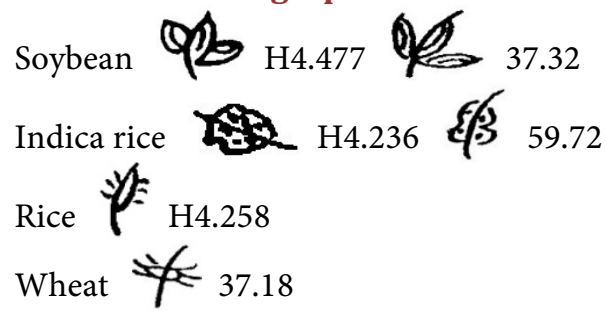

3.2.2. Partial Pictograph on Bird

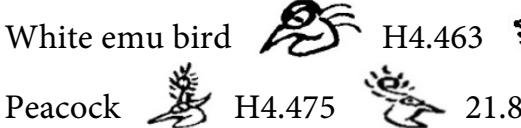

\subsubsection{Partial Pictograph on Animals}

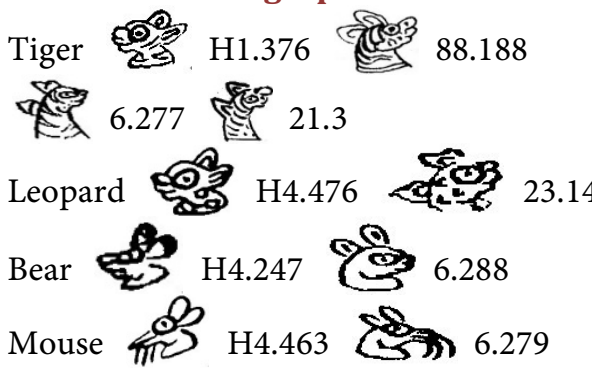




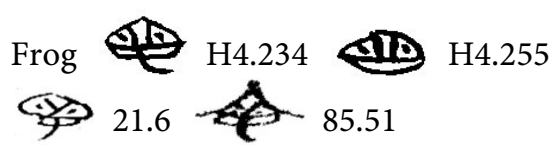

The partial pictograph in Lijiang area is mainly focus on plants, birds and animals, for this kinds of object has the individuality, so taking the partial part to create partial pictograph can distinguish with other forms, and can representative the exact meaning.

\section{Compound Pictograph of Dongba Script in Lijiang}

Compound pictograph of dongba script is composed by several parts, expressing an integral meaning together. Compound pictograph can be divided into the same body compound pictograph and the different body compound pictograph.

\subsection{The Same Body Compound Pictograph}

\subsubsection{Star $\bigcirc \mathrm{H} 4.471-0^{\circ}$ o $\mathrm{H} 4.233$}

The meaning of the scripture in Figure 5 is "The great God heng-yi-ge-kong bestows big power". $0\left[\mathrm{kw}^{21}\right]$ "star", here is borrowed to represent the third syllable of "heng-yi-ge-kong".

The meaning of the scripture in Figure 6 is "Ancient times, the sky is covered with stars, and today's stars are lucky stars. Grass covers on the ground and today's grass are the greenest." $0^{\circ} \circ$ is the plurality of the $\boldsymbol{O}$, representing the group of stars.

Sometimes there will be some dots around the script $0^{\circ} \circ$, also representing the meaning of group of stars. For example:

The meaning of the scripture in Figure 7 is "May my children and grandchildren as more as stars, countless." Adding many dots around $0^{\circ} \circ$ refers to group of stars.

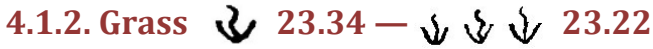

The meaning of the scripture in Figure 8 is "Stuck grass puppet into the cattle's mouth, and stuck the wood and stone into cliff hole."

The meaning of the scripture in Figure 9 is "there are 'duo' ghost of white

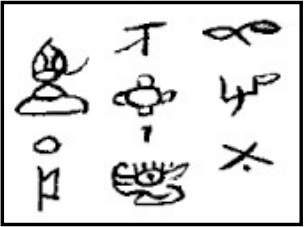

Figure 5. H4.471.

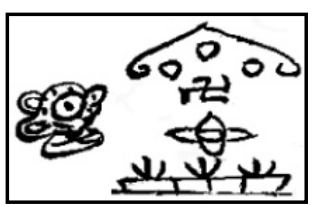

Figure 6. H4.233. 


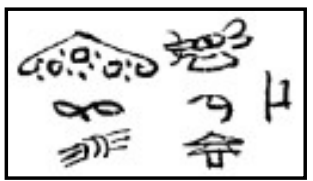

Figure 7. H4.257.

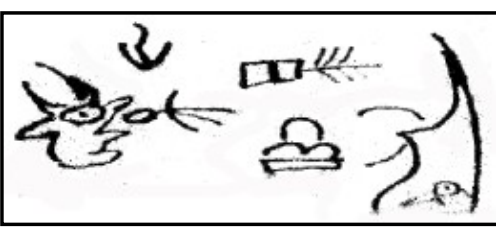

Figure 8. 23.34.

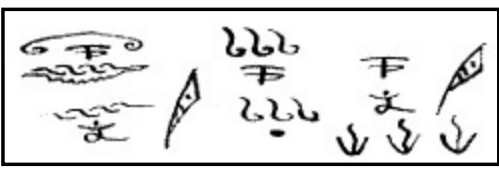

Figure 9. 23.22.

cloud and black cloud in the sky. The white cloud and black cloud change to white dew and black dew, bringing about the 'duo' ghost of white dew and black dew. The white dew and black dew fall onto the white grass and black grass, bringing about the 'duo' ghost of white grass and black grass."

The dependent script 2 is used in 23.34 to express the meaning of grass, and the compound pictograph $\downarrow \mho \downarrow$ is used in 23.22 to express the same meaning of grass, both of them appearing in the same scripture, written by the same dongba.

\subsection{The Different Body Compound Pictograph}

\subsubsection{Hair 逍 23.34}

The meaning of the scripture in Figure 10 is "buried the scattered black hair in the black soil, buried golden shoes in the earth and stone, then step on it." 数 $\left[\mathrm{fv}^{33}\right]$ hair, the script is just the shape of the hair on the head, maybe dongba consider just hair may confused with other things, so he writes out the head, which is attached pictograph.

Actually, there is the scripture, expressing the meaning of hair, just writing the hair, for example:

The meaning of the scripture in Figure 11 is "people cannot talk, and they just say ambiguous words; trees cannot grow, and they just grow on the bending area". You can see in this scripture, the character $\leqslant$. just writes the shape of the hair.

\section{The Features of Lijiang Dongba Pictograph Script}

\subsection{Large Number and High Level of Pictogram}

In Lijiang dongba script, pictograph occupies the absolute dominant position. 


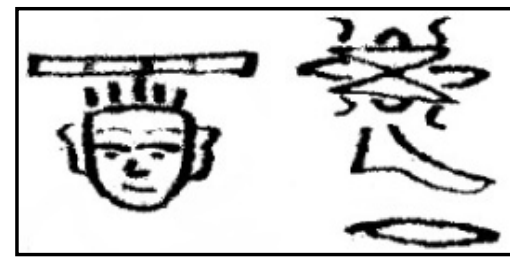

Figure 10. 23.34.

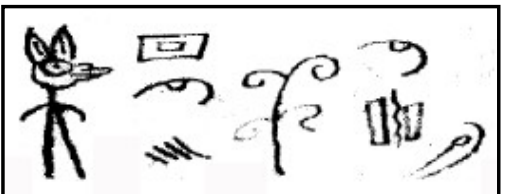

Figure 11. 23.39.

We research on ten dongba scriptures, and make a dongba text form. The form collects 574 dongba scripts, and collects 380 pictograph characters, $66.2 \%$ of which are pictograph characters. Pictograph is the main configuration form of Lijiang dongba.

Also Lijiang dongba script enjoys a high level of Pictograph of writing.

\subsection{More Partial Pictograph}

Relative to the overall pictograph, the number of partial pictograph of Lijiang dongba script, is larger, especially the animal characters.

We do a statistics in the scripture < throw ka-lv Flour doll > and find 25

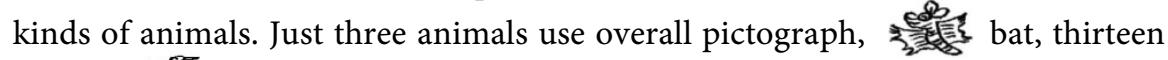
times; insect, two times. Twenty-two animals use partial pictographic, teen times; three times, and so on.

According to the research, we can find the animal characters in Lijiang dongba use more partial pictographs. Relatively speaking, the overall pictograph uses less. On one hand, writing partial pictographs is more convenient and managing; on the other hand, some animal characters are mostly used for borrowing the pronunciation. For an animal character that is mostly used for borrowing, there is no need to spend a lot of time and energy on the overall pictograph.

Therefore, on the premise that the same can be expressed accurately, the superiority of the partial pictograph is demonstrated.

\section{Conclusion}

Pictograph is the most important feature and the most important script-making method of dongba script. Through the study above, we know more content and features about the pictograph in dongba script. Both of the overall pictograph and the partial pictograph are usual and important types to express the meaning. Besides them, if there are other types of pictograph to express the meaning, it 
still needs further study.

\section{Funding}

This article is sponsored by Chongqing Social Science Fund project (Item Number: 2015BS008); Chongqing Postdoctoral Special Fund project (Item Number: Xm2017153); Chinese Postdoctoral Science Fund project (Item Number: 2016M602621).

\section{References}

[1] Li, X. (2004) Naxi Pictograph. Yunnan Renming Press, Kunming.

[2] Fang, G.Y. and He, Z.W. (2005) Word Spectrum of Naxi Pictograph. Yunnan Renming Press, Kunming, 42-44.

[3] Li, L.C. (1984) The Mo-So Classic Collected in Library of Congress. In: Li, L.C., Ed., The Theses of Mo-So Study, Taiwan Palace Museum Press, Taiwan, 127-160.

[4] The Institute of Domba Culture. (1999) The Collected Works of Naxi Domba Manuscripts. Yunnan Renming Press, Kunming.

[5] Institute of Ethnology and Anthropology, Chinese Academy of Social Sciences. (2011) Naxi Dongba Manuscripts Collected by Harvard-Yenching Institute. China Social Sciences Press, Beijing.

[6] Yu, S.S. (2003) Study on Naxi Dongba Pictographs. In: Yui, S.S., Ed., The Theses of Naxi Domba Character Study, Bashu Shushe Press, Chengdu, 235-251. 